\title{
On-Chip Cellomics for Cardiotoxity: Cell Network Model For Re-Construction of Higher Complexity of Organs
}

\author{
Kenji Yasuda*, Tomoyuki Kaneko and Fumimasa Nomura
}

Institute of Biomaterials and Bioengineering, Tokyo Medical and Dental University, 2-3-10 Kanda-Surugadai, Chiyoda, Tokyo 101-0062, Japan

\begin{abstract}
Cells are minimum units reflecting epigenetic information, which is considered to map the history of a parallelprocessing recurrent network of biochemical reactions, their behaviors cannot be explained by considering only conventional DNA information-processing events. We have developed methods and systems of analyzing epigenetic information in cells, as well as that of genetic information, to expand our understanding of how living systems are determined. The role of epigenetic information on cells, which complements their genetic information, was inferred by comparing predictions from genetic information with cell behaviour observed under conditions chosen to reveal adaptation processes and community effects. A system of analyzing epigenetic information was developed starting from the twin complementary viewpoints of cell regulation as an 'algebraic' system (emphasis on temporal aspects) and as a 'geometric' system (emphasis on spatial aspects). The knowlege acquired from this study may lead to the use of cells that fully control practical applications like cell-based drug screening and the regeneration of organs. As one of the practical application, we have developed the on-chip cardiotoxity measurement system for monitoring the risk of the lethal ventricular arrhythmia Torsade de pointes (TdP), which is the most common reason for the withdrawal or restricted use of many cardiovascular and non-cardiovascular drugs. The lack of an in vitro model to detect pro-arrhythmic effects on human heart cells hinders the development of new drugs. We also exploited the recently established human induced pluripotent stem (hiPS) cells driven to differentiate into functional cardiomyocytes. The hiPS-derived cardiomyocytes (hiPS-CMs) were analyzed using our on-chip cardiotoxity measurement system. The application of ion channel inhibitors resulted in dose-dependent changes to the field potential waveform, and these changes were identical to those induced in the native cardiomyocytes. This study shows that hiPS-CMs represent a promising in vitro model for cardiac electrophysiologic studies and drug screening.
\end{abstract}

Keywords: Epigenetic, Torsade de pointes (TdP), cardiomyocytes, Multiple electrode arrays (MEAs), Human induced pluripotent stem (hiPS).

\section{INTRODUCTION}

In the 20th century, advances in knowledge about living organisms has increased dramatically and has produced the modern disciplines of genomics and proteomics. However, despite these advances, a great challenge remains in learning how the different living components of a cell are integrated and regulated. As we move into the post-genomic period, the complementarity of genomics and proteomics will become more apparent, allowing the connections between them to be exploited. However, neither genomics nor proteomics based only on genomic information can provide the knowledge needed to interconnect molecular events in living cells. The cells in a group are individual entities, and differences arise even among cells with identical genetic information that have grown under the same conditions. These cells respond differently to perturbations [1]. Why and how do these differences arise? Cells are minimum units determining their responses through genetic and epigenetic information like the history of interactions between them and fluctuations in environmental conditions affecting them. To understand the

*Address correspondence to this author at the Institute of Biomaterials and Bioengineering, Tokyo Medical and Dental University, 2-3-10 KandaSurugadai, Chiyoda, Tokyo 101-0062, Japan; Tel: +81-3-5280-8046; Fax: +81-3-5280-8049; E-mail: yasuda.bmi@tmd.ac.jp rules underlying possible differences occurring in cells, we need to develop methods of simultaneously evaluating both the genetic and epigenetic information. In other words, if we are to understand adaptation processes, community effects, and the meaning of cell network patterns, we need to analyze their epigenetic information. We thus started a project focusing on developing a system that could be used to evaluate the epigenetic information in cells by continuously observing specific examples and their interactions under controlled conditions. The importance of understanding epigenetic information is expected to become apparent in cell-based biological and medical fields like cell-based drug screening and the regeneration of organs from stem cells, fields where phenomena cannot be interpreted without taking epigenetic factors into account.

We started a study on the "determination of genetic and epigenetic control processes in cells" using on-chip microfablication techniques and cell-based analysis. To understand the meaning of genetic information and epigenetic correlation in cells, we developed an on-chip single-cell-based microcultivation method. As we can see in Fig. (1), the strategy behind our method is constructive, involving three steps. First, we purify cells from tissue one by one in a nondestructive manner [2]. We then cultivate and observe them under fully controlled conditions (e.g., cell 


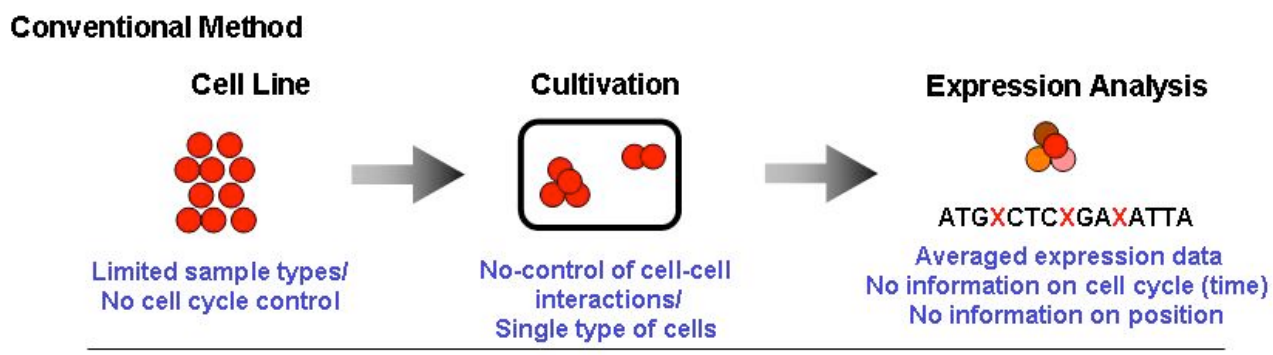

\section{Our Method}
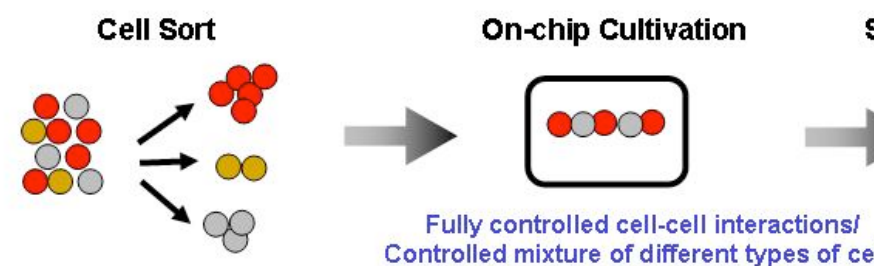

Single-cell Expression Analysis

Separation of different types of cells

No limit on cell types

No-need for precultivation

Fig. (1). Our strategy: on-chip single-cell-based analysis.

population, network patterns, or nutrient conditions) using an on-chip single-cell cultivation chip [3-12] or an on-chip agarose microchamber system [13-20]. Finally, we do single-cell-based expression analysis through photothermal denaturation and single-molecule level analysis [21]. In this way, we can control the spatial distribution and interactions of cells.

\section{WHY ON-CHIP TECHNOLOGY?: CULTIVATION SYSTEMS FROM 'ALGEBRAIC' AND 'GEOMETRIC' VIEWPOINTS}

The first aim of our single-cell-based study is to develop methods and systems that enable the mechanism responsible for controlling (regulating) cells epigenetically to be analyzed. It is based on the idea that, although genetic information creates a network of biochemical reactions, its history as a parallel-processing recurrent network was ultimately determined by the environmental conditions of cells, which we call epigenetic information. As previously discussed, if we are to understand the events in living systems at the cellular level, we need to bear in mind that epigenetic information complements the genetic information.

The advantage of this approach is that it removes the complexity in underlying physicochemical reactions that are not always completely understood and for which most of the necessary variables cannot be measured. Moreover, this approach shifts the view of cell regulatory processes from a basic chemical ground to a paradigm of the cell as an information-processing unit working as an intelligent machine capable of adaptating to changing environmental and internal conditions. This is an alternative representation of the cell and can bring new insights into cellular processes. Thus, models derived from such a viewpoint can directly help in more traditional biochemical and molecular biological analyses that assist in our understanding of control in cells.

The main purpose of the study was to develop on-chip single-cell-based cultivation and analysis systems to monitor dynamic processes in the cell. We have used these systems to extend ideas from the genetic to the genetic-epigenetic network in investigating topics like variations in cells with the same genetic information, inheritance of non-genetic information between adjacent generations of cells, cellular adaptation processes caused by environmental change, the community effect of cells, and network pattern formation in cell groups (Figs. (2) and (3)). After sufficient experimental observations, we can understand the role of epigenetic information in modeling more complex signaling cascades. This field has almost been entirely monopolized by physicochemical models, which provide a good standard for comparison, evaluation, and development with our approach. The ultimate aim of our study is to provide a comprehensive understanding of living systems as products of both genetic and epigenetic information. It would permit us to describe the phenomena occurring in cell systems sufficiently well to be able to interpret and control them.

\section{CULTIVATION SYSTEM FOR 'GEOMETRIC' VIEWPOINT: ON-CHIP AGAROSE MICROCHAMBER CULTIVATION SYSTEM}

An approach to studying network patterns (or cell-cell interactions) and the community effect in cells was to create a fully controlled network by using cells on the chip (Fig. (3)).

For understanding the reaction of cells to the topography of the substratum, which occurs in the development and natural regeneration of tissue, a silicon wafer and a glass slide with holes and metal decorations have been created and tested [22-25]. Though these conventional microfabrication techniques provide structures with fine spatial resolution, it is still hard to change the shape of these structures during cell cultivation, which is usually unpredictable and is only defined during cultivation. A variety of materials and several well-known methods, including bonding, sacrificial layer techniques, and lamination have been used to create tunnelshaped microstructures between two microchambers [26]. 


\section{Questions}

- Are there any differences between cells that contain the same genomes and contents?

- Is epigenetic information inherited between the adjacent generations?

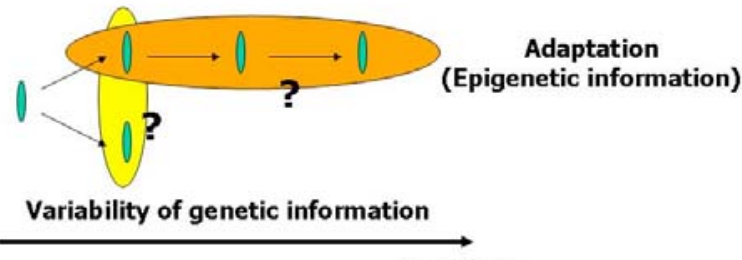

Generations

Fig. (2). Aim of the single-cell based analysis (1): temporal aspect.

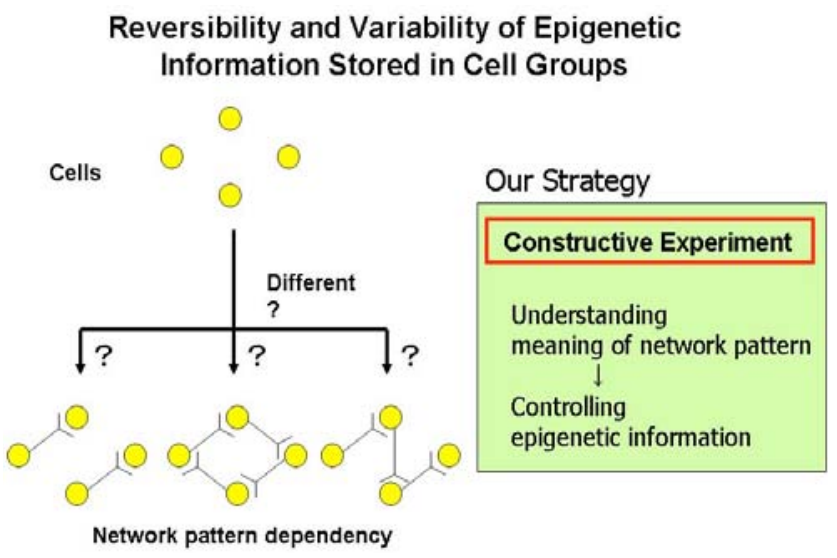

Fig. (3). Aim of single-cell-based analysis (2): spatial aspect.

Creating small tunnels to connect two chambers without cells passing through is essential in cell cultivation. However, making these microstructures on a chip still requires a number of steps, and it is impossible to do during cell cultivation.

We therefore developed a system consisting of an agarmicrochamber (AMC) array chip, a cultivation dish with a nutrient-buffer-changing apparatus, a permeable cultivation container, and a phase-contrast/fluorescent optical microscope with a 1064-nm/1480-nm focused-laser-irradiation apparatus to create photothermal spot heating (Fig. (4)) [13-
20]. The most important advantage of this system was that we could change the microstructures in the agar layer even during cultivation, which is impossible when conventional $\mathrm{Si} /$ glass-based microfabrication techniques and microprinting methods are used.

\section{Photo-Thermal Etching Method}

The agarose-microchamber cell-cultivation system has an apparatus for photothermal etching $[13,14]$. This is the areaspecific melting of agarose microchambers by spot heating using a focused laser beam of $1480 \mathrm{~nm}$, and of a thin layer made of a light-absorbing material such as chromium with a laser beam of $1064 \mathrm{~nm}$ (since agarose itself has little absorbance at $1064 \mathrm{~nm}$ ). The system has two parts: a phasecontrast microscope (IX-70; with a phase-contrast objective lens, $\times 40$, Olympus, Tokyo, Japan) with an automated X-Y stage (BIOS-201T, Sigma Koki, Hidaka, Saitama, Japan), and a dual wavelength focused-laser-irradiation module with a 1064-nm Nd:YAG laser (max. 1 W; Forte-1064, Laser Quantum, Emery Court, Vale Road, Stockport, Cheshire, UK) and a 1480-nm Raman fiber laser (max. 1 W; PYL-110480-M, IPG Photonics, Oxford, MA, USA). For phasecontrast microscopy and $\mu \mathrm{m}$-scale photo-thermal etching, three different wavelengths (visible light for observation and 1480-nm/1064-nm infrared lasers for spot heating) were used simultaneously to observe the positions of the agar chip surface and to melt a portion of the agar in the area being heated. A phase-contrast image was acquired by using a charge-coupled device (CCD) camera (CS230, Olympus). The dichroic mirrors and lenses in the system were chosen for these three different wavelengths. Flexible-slide focusing lenses were placed in the path of the infrared laser beam to control the focal positions of the lasers to correct their different focal lengths, which depended on the wavelengths.

We used a new type of non-contact three-dimensional photo-thermal etching in this system for the agarmicroetching to exploit the characteristics of the two different infrared laser beam wavelengths (1480 and 1064 $\mathrm{nm})$. As the 1480-nm infrared beam was absorbed by water and agar gel, the agar gel in the light pathway was heated and completely melted. As the 1064-nm infrared beam, on the other hand, did not have this absorbance, the agar melted just near the thin chromium layer, which absorbed the beam.

Using this non-contact etching, we could easily produce microstructures such as holes and tunnels within only a few

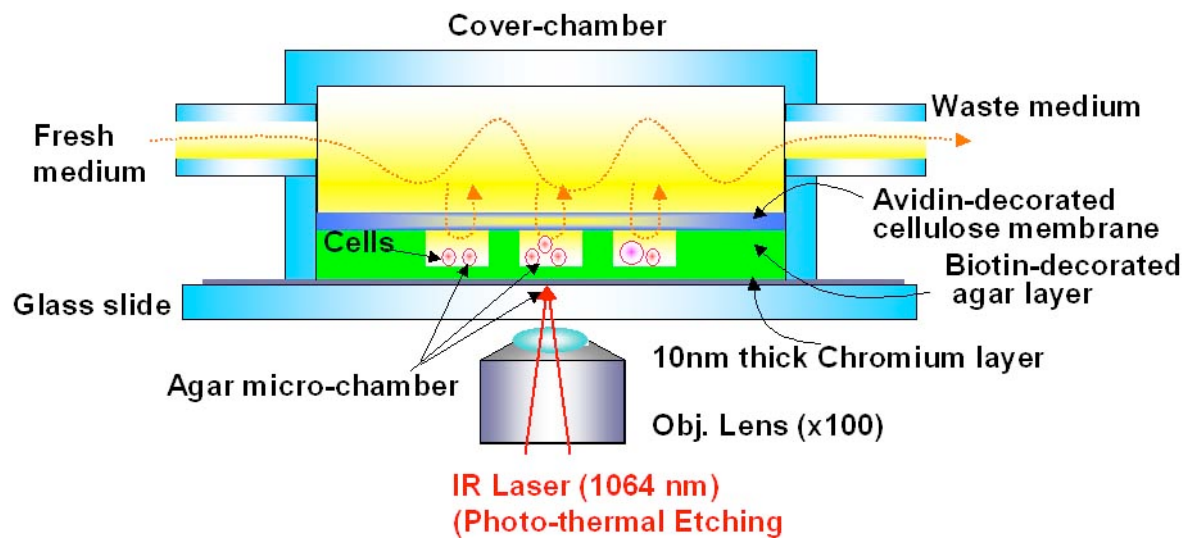

Fig. (4). Agarose microchamber cell cultivation system with photo-thermal etching unit. 

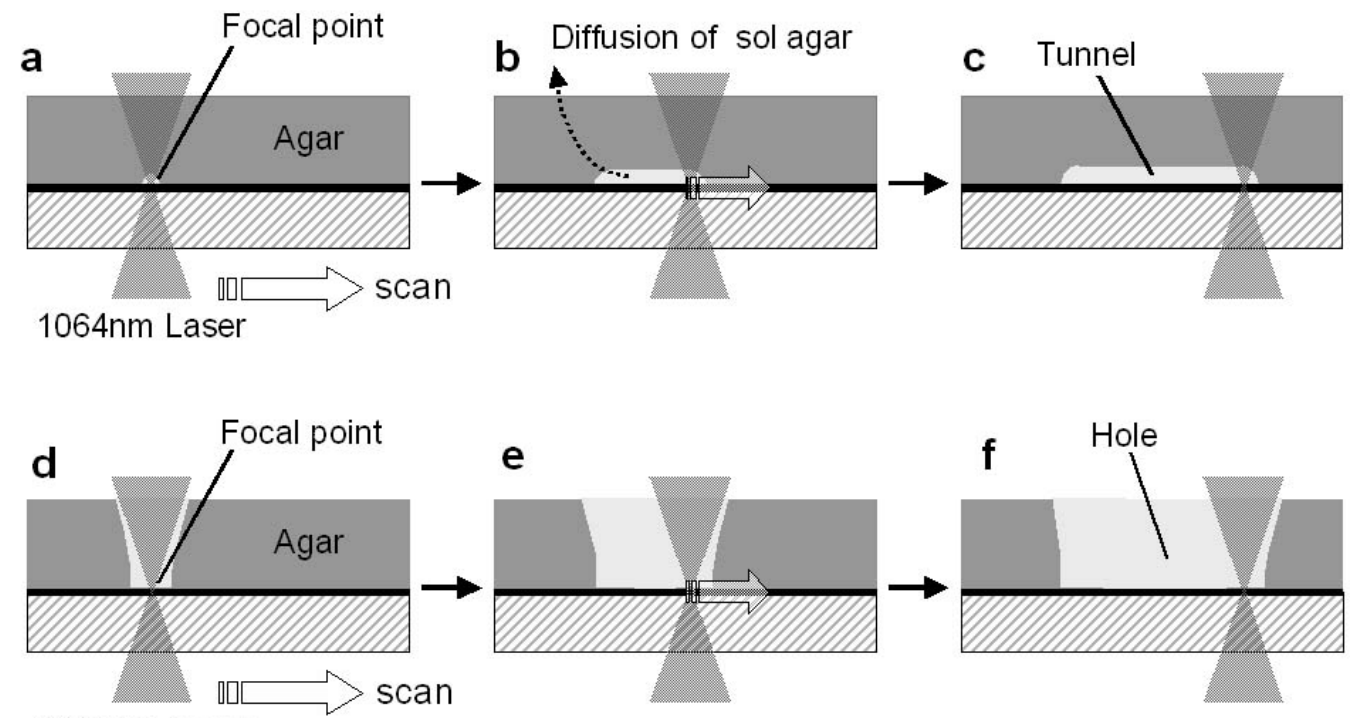

1480nm Laser

Fig. (5). Photo-thermal etching method: (a)-(c), $1064 \mathrm{~nm}$; (d)-(f), $1480 \mathrm{~nm}$.

minutes. As we can see from Fig. (5), the laser melted the agar as follows. (a) When a 1064-nm infrared laser beam was focused on the chromium layer on the glass slide, the agar at the focal point near the chromium layer started to melt. (b) Then, when the focused beam was moved parallel to the chip surface, a portion of the agar at the heated spot melted and diffused into the water through the agar mesh. (c) After the heated spot had been moved, a tunnel was created at the bottom of the agar layer. (d) However, when a 1480$\mathrm{nm}$ infrared laser beam was focused on the agar glass slide, the agar in the light path started to melt. (e) When the focused beam was moved parallel to the chip surface, a portion of the agar in the light path melted and diffused into the water. (f) Finally, after the heated spot had been moved, a hole was created on the glass slide.

We made a three-dimensional structure for the agar microchambers with a photo-thermal etching module. Fig.
(6) is a top-view micrograph of the agar microchambers connected by small channels. The space on the chip was colored by filling the microchambers with a fluorescent dye solution. Also shown are cross-sectional views of the A-A and $\mathrm{B}-\mathrm{B}$ sections, where we can easily see narrow tunnels under the thick agar layer in the A-A section and round tunnels in the B-B section. These cross-sectional micrographs reveal that we can make narrow tunnels in the agar layer with photothermal etching. The left micrograph in Fig. (6) is a top view of the entire microchamber array connected by narrow tunnels.

\section{Controlling Cell Network Pattern with Photo-thermal Etching}

In the field of neuroscience, one of the main interests of epigenetic study is how the epigenetic information is processed and recorded as plasticity within a network
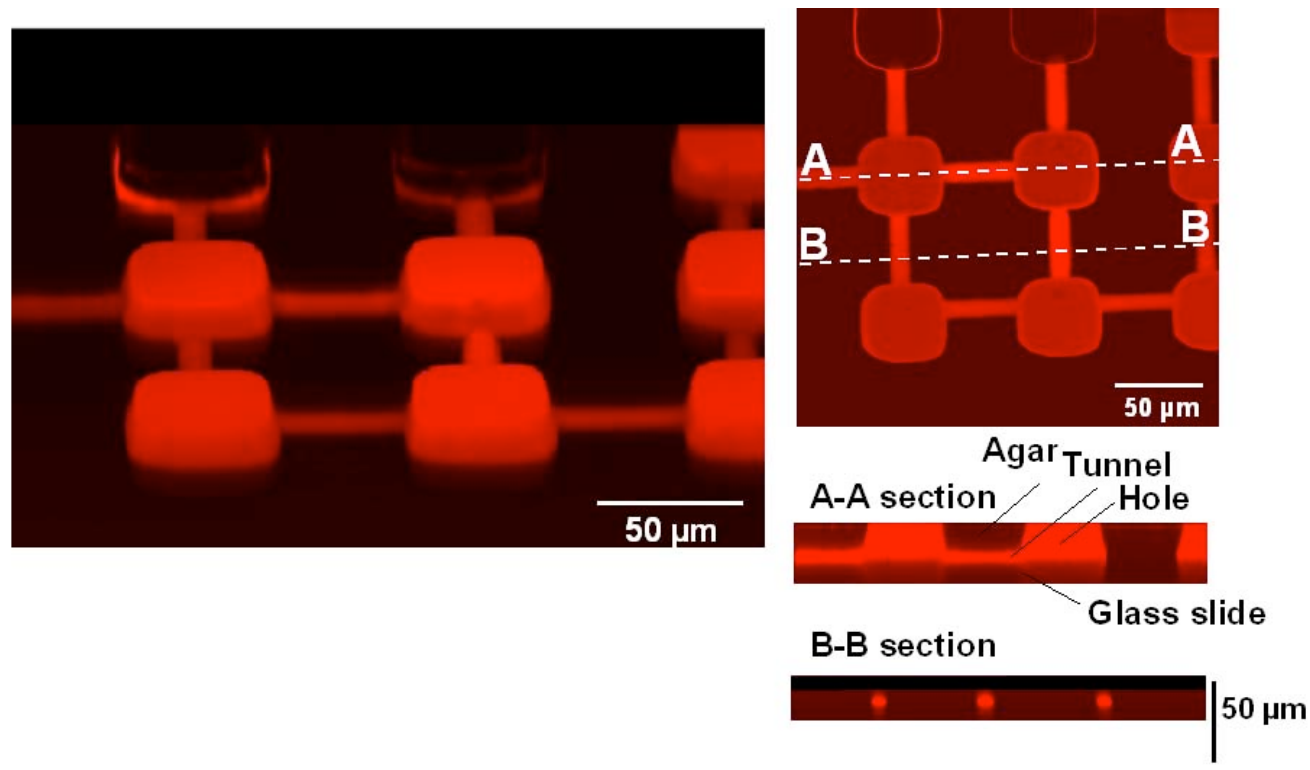

Fig. (6). Agarose microchambers fabricated with photo-thermal etching. 
pattern, what might be caused by the change in the network pattern or by the degree of complexity related to the network size. To understand the meaning of the network pattern and size, one of the best approaches is to analyze the function of an artificially constructed neural cell network under fully controlled conditions. Thus, for many years, neurophysiologists have investigated single-cell-based neural network cultivation and examined the firing patterns of single neurons through the fabrication of cultivation substrates using microprinting techniques [27-29], patterning on silicon-oxide substrates [30], and three-dimensional structures made using photolithography [31]. Although these conventional microfabrication techniques provide structures with fine spatial resolution, effective approaches to studying epigenetic information are still being sought. With conventional techniques, it is still hard to make flexible microstructures with simple steps or to change their shape during cultivation since the shape is usually unpredictable and only defined during cultivation.

Non-invasive extracellular measurement using a multielectrode array (MEA) has been in use since the 1970s [3241], and has proven to be an effective long-term electrophysiological measurement technique for neural cells. Advances in microfabrication technologies have enabled the creation of an electrode array that can be used to simultaneously observe the firing of multiple cells, but the problems of contamination and cells escaping from the position of each electrode remain and often occur in longterm cultivation.

Thus, we have developed a new single-cell cultivation system using agarose microstructures and a photo-thermal etching method $[13,14]$. Using the photo-thermal etching method, we can form microstructures within the agarose layer on the chip by melting a portion of the agarose layer at the spot of a focused infrared laser beam as described above.
This method can be applied even during cultivation, so we can change the network pattern of nerve cells during cultivation by adding microchannels between two adjacent microchambers in a step-by-step fashion [15-18]. This helps us understand the meaning of the spatial pattern of a neuronal network by comparing the changes in signals before and after the network shape is changed. Moreover, we developed an agarose microchamber (AMC) system on an MEA substrate that can be used to obtain the long-term electronic properties of topographically controlled neuronal networks with precise fixation of cell positions and flexible network pattern rearrangement through photo-thermal etching of the agarose layer. In this subsection, we describe our newly developed neural-cell cultivation chip and its cultivation/recording system [15-18].

Applying stepwise photo-thermal etching to an AMC array during cultivation, we have developed a method of controlling the topography in the direction of synaptic connections in the network patterns of a living neuronal network [18]. This allowed the direction in which axons were elongated to be flexibly controlled by melting the narrow micrometer-order grooves (microchannels) in steps through photo-thermal etching where a portion of the agarose layer was melted with the 1064-nm infrared laser beam. Fig. (7) shows an example of this procedure. The micrographs are phase-contrast images of the growth of single hippocampal cells in three of the microchambers. When cultivation started, single cells were placed into the AMCs (Fig. (7a)). Six hours later, single neurites elongated from the cells into the microchannels (Fig. (7b)). At that time, because we found that the elongation of neurites was sufficiently stable, additional photo-thermal etching were done to connect two adjacent AMCs (Fig. (7c)). Two hours after the additional photo-thermal etching (8-h cultivation), all three cells retained their shapes and continued the a

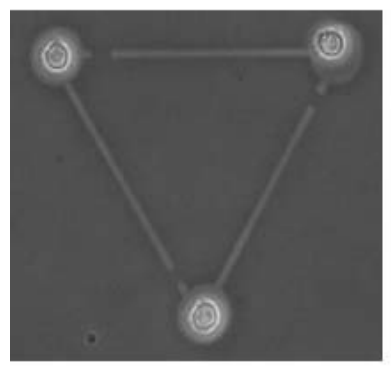

d

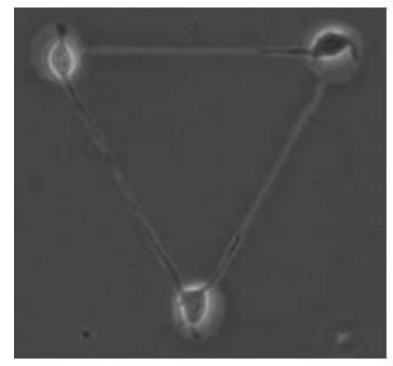

b

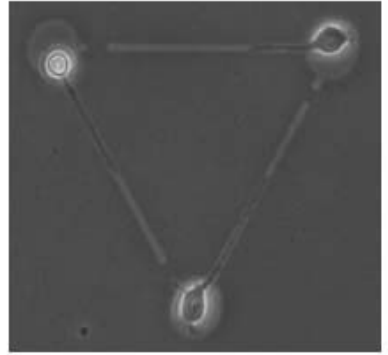

e

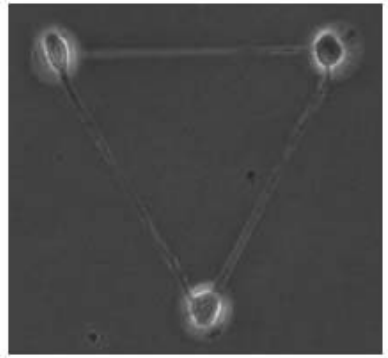

C

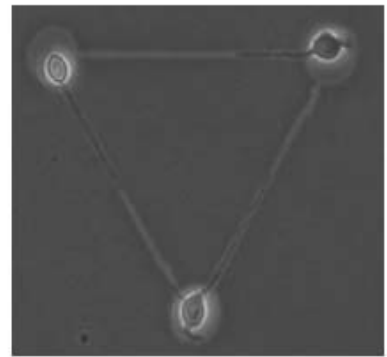

f

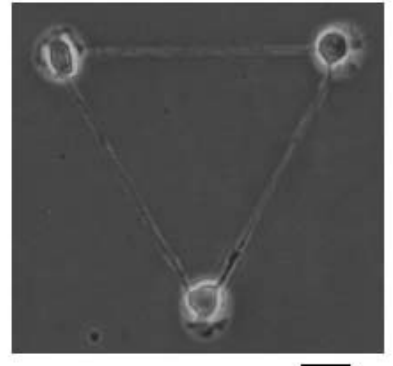

Fig. (7). Stepwise formation of neuronal network pattern in rat hippocampal cells. 
elongation (Fig. (7d)). Finally, these three cells were connected counter-clockwise by the neurites. (Figs. (7e) and (7f), 14 and 28-h cultivation). In contrast, if we had not used stepwise photo-thermal etching, cells in microchambers with two tunnels would have elongated both clockwise and counter-clockwise simultaneously and we would not have been able to distinguish in what direction the neurites had elongated.

Using the system we previously described, we formed an individual-cell-based neural network pattern of rat hippocampal cells within the AMC array, without the cells escaping, and controlled the direction axons were elongated by using stepwise photo-thermal etching during cultivation. This demonstrated the potential of our on-chip AMC cell cultivation system in single-cell-based direction-controlled neural network measurement.

Moreover, we developed a new type of individual-cellbased electrophysiological measurement using an on-chip multi-electrode array (MEA) cell-cultivation system with an AMC array to control the topography of network patterns in a living neuronal network [17]. The advantages of this MEA system are that it allows multiple cells firing simultaneously to be recorded for weeks without contamination, and that it allows cell positions, numbers, and their connections for cultivation to be controlled using AMCs with microchannels fabricated by photothermal etching where a portion of the agarose layer is melted with a 1480-nm infrared laser beam. Using this method, we formed an individual-cell-based neural network pattern of rat hippocampal cells within the AMC array without cells escaping from electrode positions in the microchamber during a thirteen-day cultivation period (Fig. (8)).

\section{Synchronization Dynamics in Cardiac Myocyte Networks}

The agarose microchamber system can also be used to observe the dynamics of the synchronizing process for two

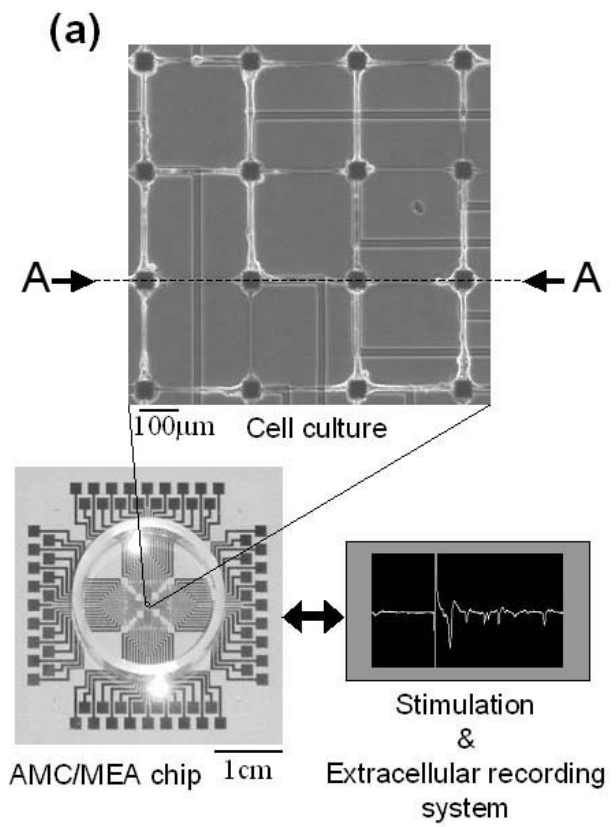

isolated rat cardiac myocytes (Fig. (9)) [19]. After cultivation had started, the two cells elongated and made physical contact within 24 hours. They then synchronized. It should be noted that (see graph) the synchronization process involved one of the cells following the other, and that, before synchronized beating started, the cell that ended up following stopped beating to synchronize the timing. Using this method, the number and spatial distribution of cardiac myocytes can easily be controlled (Fig. (10)) [20]. For example, the fluctuation of pulsation intervals was reduced when the number of the connected cardiac myocyte cells was increased. It demonstrates one example of non-linear community effect of cell group for stabilizing the rhythm against the environmental change.

\section{On-Chip In Vitro Pharmacologic Testing Using Human Induced Pluripotent Stem Cell-Derived Cardiomyocytes}

Torsade de pointes (TdP), which is a polymorphic ventricular tachycardia that can degenerate into ventricular fibrillation and subsequent cardiac sudden death, may be associated with drug-induced QT prolongation. Druginduced TdP is a serious issue in drug development and safety, and has led to the withdrawal of many noncardiovascular drugs. Despite the rarity of TdP in the clinical setting, definition of the pro-arrhythmic potential of a candidate drug is important in terms of patient safety and drug development. There is therefore an important need for a preclinical model to predict TdP. Several in vivo and in vitro models are currently used in cardiovascular drug development, testing, and screening for side-effects [42-44]. Inhibition of hERG, which is a rapid delayed rectifier current $\left(\mathrm{I}_{\mathrm{Kr}}\right)$ that controls action potential (AP) repolarization, is the most common cause of QT prolongation induced by noncardiac drugs. Thus, non-clinical evaluation of the potential for delayed ventricular repolarization must be conducted using the hERG assay [45].

(b)

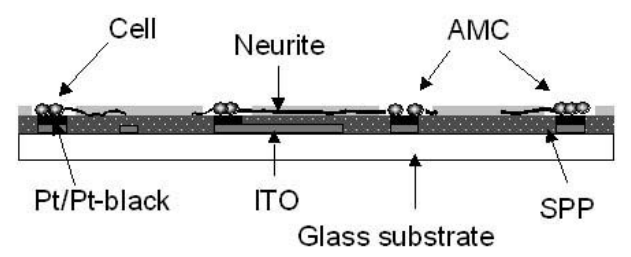

Fig. (8). Micrographs and schematic drawings of on-chip AMC/MEA cell cultivation chip. (a) Phase-contrast micrograph of AMC/MEA chip. Agarose microchambers and microchannels connecting them were fixed on substrate of metal electrode arrays. (b) Schematic drawing of cross-sectional view of A-A section of chip in (a) 


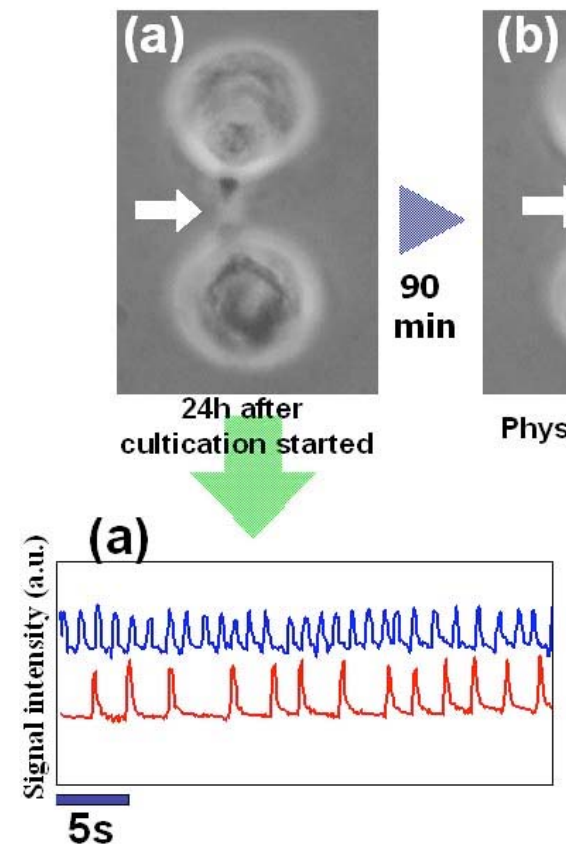

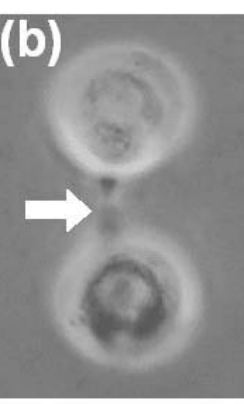

Physical contact
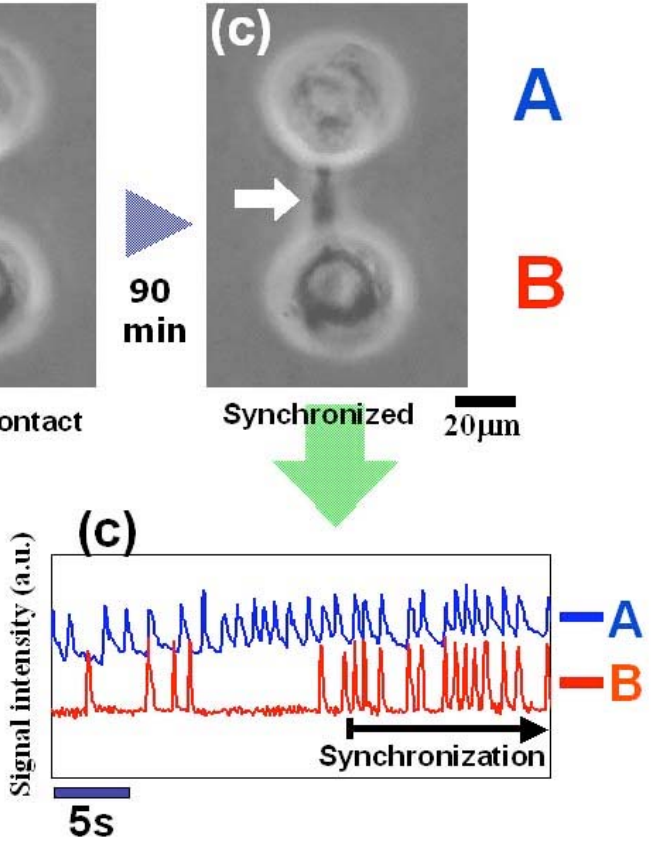

Fig. (9). Synchronization of two cardiac myocyte cells.

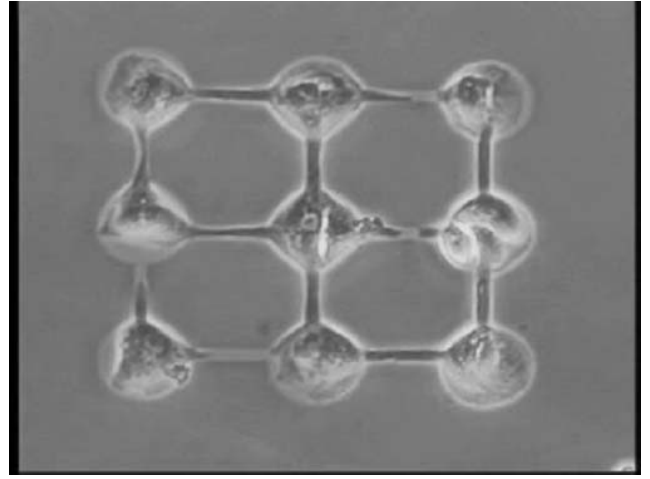

Fig. (10). Topographical control of number of cardiac myocyte cells and connections.

Induced pluripotent stem (iPS) cells were recently established by transfecting murine and human fibroblasts with the transcription factors Oct3/4, Sox2, Klf4, and c-Myc, which are expressed at high levels in embryonic stem (ES) cells [46-48]. In regard to drug discovery, hiPS cells should make it easier to generate panels of cell lines that more closely reflect the genetic diversity of a population. As there are several genetic disorders known to affect the heart, such as familial cardiomyopathy, familial lethal arrhythmias, and congenital heart diseases, it is necessary to examine the development, pathogenesis, and physiologic characteristics of the hereditarily diseased human cardiomyocytes. In these respects, hiPS-derived cardiomyocytes (hiPS-CMs) confer significant advantages, since it is difficult to obtain living diseased cells from patients for the purposes of drug screening and analyses. In neurodegenerative diseases such as amytrophic lateral sclerosis, Parkinson disease, and spinal muscular atrophy, patient-specific iPS cells have already been established [49-51]. To date, there has been no report on the establishment of in vitro models using hiPS-CMs for drug screening.

Multiple electrode arrays (MEAs) enable measurement of the surface electrogenic activities of cell clusters. MEAs are easy to operate and can be adapted to automatic highthroughput systems. In addition, MEAs permit stable and long-duration recordings, which are necessary to evaluate the relationships between dose-dependency and the induction of side-effects for new drugs. The washing out of drugs from the external solution leads to the recovery of the field potential (FP) waveform almost to the baseline observed before drug application, which means that this system can be used to test the reproducibility of pharmacologic effects and that the effects of several drugs can be examined using the same preparation. In this section, we introduce a set of experiments that we have examined the electrophysiologic capacity of hiPS-CMs in responding to several cardiac drugs and measured the responsiveness of hiPS-CMs to representative sodium, calcium, and potassium ion channel blockers. This is the first report to validate the concept of drug screening using hiPS-CMs together with the MEA system.

\section{Drug Screening Using hiPS-CMs Together with the On- Chip MEA System}

The hiPS cell line 201B7, into which Oct3/4, Sox2, Klf4, and c-Myc were retrovirally transfected [46], was maintained in the undifferentiated state by co-culturing with a mitomycin C-inactivated mouse embryonic fibroblast (MEF) feeder layer in DMEM/F12 medium (Invitrogen) that was supplemented with 20\% Knock-out Serum Replacement (KSR), $1 \mathrm{mM}$ L-glutamine, $1 \mathrm{mM}$ nonessential amino acids, $0.1 \mathrm{mM} \beta$-mercaptoethanol, and $4 \mathrm{ng} / \mathrm{ml}$ basic fibroblast growth factor (bFGF). The hiPS cell culture medium was changed daily and the cells were passaged every 5-6 days.

For embryoid body (EB) formation and cardiac differentiation, the hiPS colonies were dispersed into cell aggregates that contained 2000-5000 cells using CTK dissociation solution [52]. For differentiation, hiPS colonies 
of appropriate size were chosen using a combination of 40 $\mu \mathrm{m}$ and $100 \mu \mathrm{m}$ cell strainers (Becton-Dickinson), which also facilitated the complete removal of feeder cells. The cell aggregates were cultured in suspension in ultra-low attachment cell culture dishes in hiPS differentiation medium (DMEM/F12 supplemented with $20 \%$ FBS, $1 \mathrm{mM} \mathrm{L-}$ glutamine, $1 \mathrm{mM}$ nonessential amino acids, and $0.1 \mathrm{mM} \beta$ mercaptoethanol). The EBs were incubated at $37^{\circ} \mathrm{C}$ in $5 \%$ $\mathrm{CO}_{2}$. During differentiation, the medium was replaced every 3-4 days. Some of the EBs began to beat spontaneously 1415 days after differentiation. The beating EBs were selected for analysis.

For the field potential recordings using the on-chip MEA system, MEA chips obtained from Photo Precision (Japan), ULVAC (Japan) and Alpha-MED Science (Japan) were coated with collagen type I-C (Nitta Gelatin, Osaka, Japan) or fibronectin (Sigma). Beating EBs were plated on the electrodes and incubated at $37^{\circ} \mathrm{C}$ in a humidified atmosphere of $95 \%$ air and $5 \% \mathrm{CO}_{2}$ after the drug assay. Field potential (FP) recordings of the beating EBs were performed using the On-Chip MEA system at a sampling rate of $10-100 \mathrm{kHz}$ with low path filter of $2-10 \mathrm{kHz}$ and high path filter of $0.1-100$ $\mathrm{Hz}$, and amplified by 100-50,000 using the NF amp (NF, Japan) [53]. All MEA measurements were performed at $37^{\circ} \mathrm{C}$. The signals were initially processed with the CellAD 2.0 software (DITEC, Japan), and the obtained data were subsequently analyzed with the OriginPro 8.0J and FlexPro 7.0 programs. Data for analysis were extracted from $2 \mathrm{~min}$ to 5 min of the obtained data.

E-4031, verapamil, quinidine, and isoproterenol (all from Sigma) were prepared as $0.1 \mathrm{mM}, 1 \mathrm{mM}, 10 \mathrm{mM}$, and $10 \mu \mathrm{M}$ stock solutions in distilled water, respectively. These stock solutions were diluted to the required concentration in culture medium and applied to the test chip.

The protocol for the drug assay is described below. The medium on the test chip was exchanged for fresh medium, and then the FPs were recorded for $5 \mathrm{~min}$. Subsequently, the drug was applied to the medium at $1-5 \%$ dilution and the FPs were measured for 5 to $10 \mathrm{~min}$. Finally, washing out was performed, to exchange the medium three times per min, and 60-90 min after washout, the FPs were recorded for $5 \mathrm{~min}$.

\section{Measurement of Functional Ion Channels in hiPS-CMs Using Chemical Compounds}

To characterize the electrophysiological properties of hiPS-CMs, the FPs of the spontaneously beating EBs were recorded using the On-Chip MEA system, as previously described [53]. First, we investigated the effects of ion channel-specific inhibitors on the FP waveform, to determine whether the expressed channels were functional. As shown in Fig. (11a), the sodium channel inhibitor quinidine dosedependently and reversibly attenuated the length of time required for the decline in voltage from the baseline to the first negative peak (FP amplitude), which reflects the AP upstroke, without significant changes in the spontaneous beating rate. These findings indicate that a functional sodium channel is expressed in the hiPS-CMs. Next, we tested the effect of the calcium channel blocker verapamil on the FP waveform. Verapamil dose-dependently shortened the length of time from the first negative peak (open triangle) to the first positive peak (closed triangle) (FPD), which was consistent with the effect of calcium channel inhibition on AP duration (Fig. (11b)). Disruption of potassium channels might lead to prolongation of cardiac repolarization, potentially leading to life-threatening arrhythmias, e.g., TdP and ventricular tachycardia. Therefore, we examined the effect of the $\mathrm{I}_{\mathrm{Kr}}$ blocker E-4031 on the FP waveform. E-4031 dose-dependently and reversibly prolonged the FPD (Fig. (11c)). These results suggest that the main cardiac deporalizing and repolarizing ion channels are functionally expressed in the hiPS-CMs.

Drug-induced prolongation of the electrocardiographic QT interval is associated with an increased risk of a serious
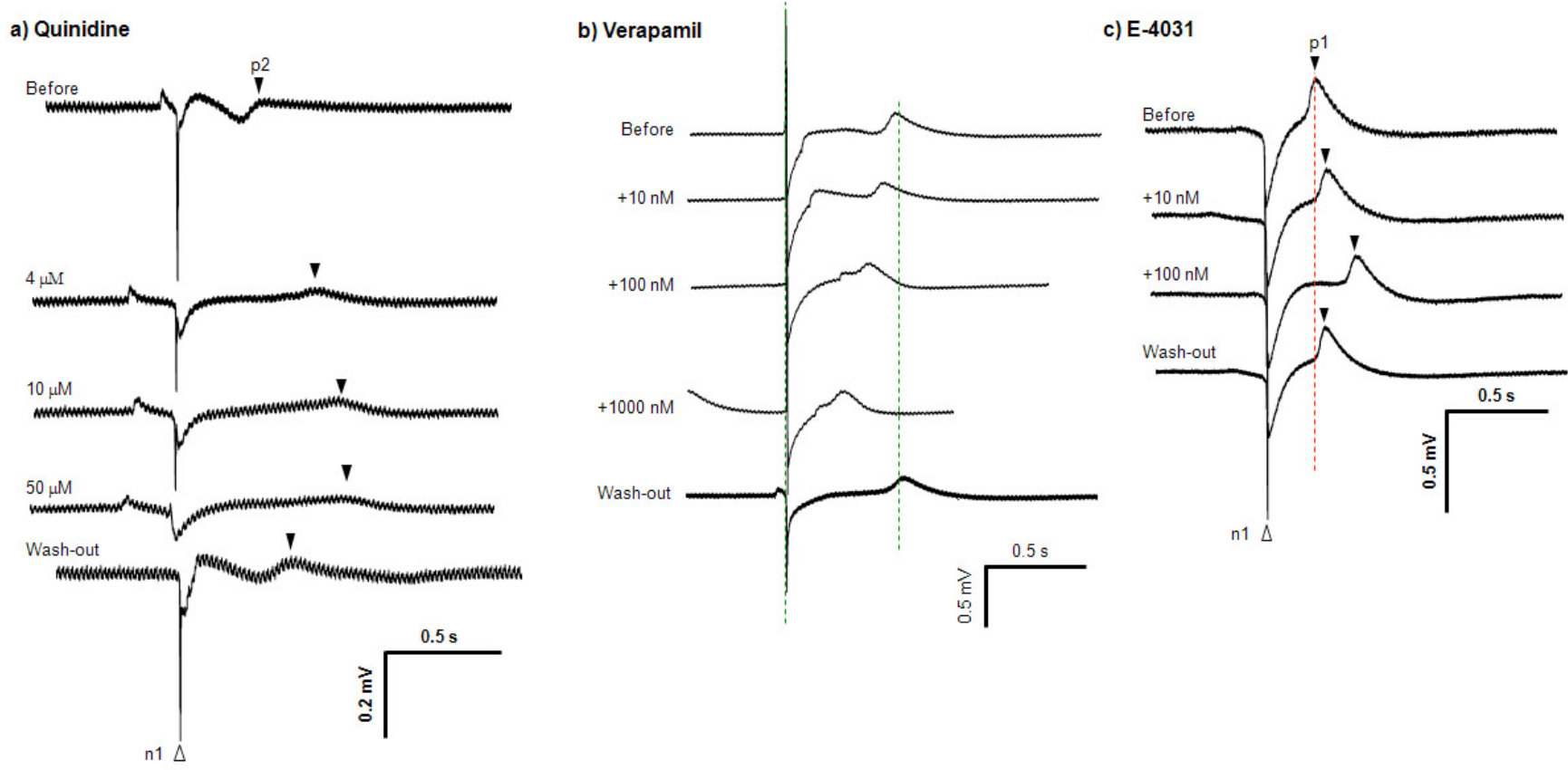

Fig. (11). Effects of quinidine (a), verapamil (b), E-4031 (c) on the field potential (FP) waveform recorded for the hiPS-derived beating EBs. 
ventricular arrhythmia, such as TdP. To avoid this problem, binding assays with radiolabeled drugs and recombinant hERGs are used. However these are typically applied to the late developing candidates due to their high costs. Furthermore, using the current system, it is difficult to predict accurately the occurrence of lethal side-effects. Thus, an in vitro model for drug screening is required, to avoid the withdrawal of drugs that have been costly to develop. We established a novel in vitro drug screening model to evaluate drug-induced changes in the electrophysiological properties of hiPS-CMs. In the present study, we measured FP using the MEA system. Although FP does not allow the derivation of an absolute measure of the individual ion currents that contribute to the AP, our MEA system clearly reveals the FP waveform changes induced by several anti-arrhythmia drugs. This indicates the potential for using a combination of the hiPS-CM and MEA systems for drug testing.

\section{CONCLUSION}

We developed and used a series of new methods of understanding the meaning of genetic and epigenetic information in a life system exploiting microstructures fabricated on a chip. The most important contribution of this study was to be able to reconstruct the concept of a cell regulatory network from the 'local' (molecules expressed at certain times and places) to the 'global' (the cell as a viable, functioning system). Knowledge of epigenetic information, which we can control and change during cell lives, complements the genetic variety, and these two kinds are indispensable for living organisms. This new kind of knowlege has the potential to be the basis of cell-based biological and medical fields like those involving cell-based drug screening and the regeneration of organs from stem cells.

\section{ACKNOWLEDGEMENTS}

The author acknowledges the assistance of all members of the Yasuda Lab., and Prof. Kennchi Fukuda of Keio Univ. and all members of his group. Financial supports, in part by the Japan Science and Technology Agency (JST), Grants-inAids for Scientific Research from the Ministry of Education, Culture, Sports, Science and Technology of the Japanese government,and by the New Energy and Industrial Technology Development Organization (NEDO). are gratefully appreciated.

\section{REFERENCES}

[1] Spudich, J.L.; Koshland, D.E. Jr. Non-genetic individuality: chance in the single cell. Nature, 1976, 262, 467-471.

[2] Yasuda, K. Non-destructive, non-contact handling method for biomaterials in micro-chamber by ultrasound. Sens. Actuators B., 2000, 64, 128-135.

[3] Inoue, I.; Wakamoto, Y.; Moriguchi, H.; Okano, K.; Yasuda, K. On-chip culture system for observation of isolated individual cells. Lab Chip, 2001, 1, 50-55.

[4] Wakamoto, Y.; Inoue, I.; Moriguchi, H.; Yasuda, K. Analysis of single-cell differences by use of an on-chip microculture system and optical trapping. Fresenius J. Anal. Chem., 2001, 371, 276281.

[5] Inoue, I.; Wakamoto, Y.; Yasuda, K. Non-genetic variability of division cycle and growth of isolated individual cells in on-chip culture system. Proc. Jpn. Acad., 2001, 77B, 145-150.

[6] Wakamoto, Y.; Umehara, S.; Matsumura, K.; Inoue, I.; Yasuda, K. Development of non-destructive, non-contact single-cell based differential cell assay using on-chip microcultivation and optical tweezers. Sens. Actuators B., 2003, 96, 693-700.
[7] Umehara, S.; Wakamoto, Y.; Inoue, I.; Yasuda, K. On-chip singlecell microcultivation assay for monitoring environmental effects on isolated cells. Biochem. Biophys. Res. Commun., 2003, 305, 534540 .

[8] Inoue, I.; Shiomi, D.; Kawagishi, I.; Yasuda, K. Simultaneous measurement of sensor-protein dynamics and motility of a single cell by on-chip microcultivation system. J. Nanobiotechnol., 2004, 2,4 .

[9] Takahashi, K.; Matsumura, K.; Yasuda, K. On-Chip Microcultivation Chamber for Swimming Cells Using Visualized Poly(dimethylsiloxane) Valves. Jpn. J. Appl. Phys., 2003, 42, L1104 - L1107.

[10] Hattori, A.; Umehara, S.; Wakamoto, Y.; Yasuda, K. Measurement of incident angle dependence of swimming bacterium reflection using on-chip single-cell cultivation assay. Jpn. J. Appl. Phys., 2003, 42, L873 - L875

[11] Matsumura, K.; Yagi, T.; Yasuda, K. Role of timer and sizer in regulation of Chlamydomonas cell cycle. Biochem. Biophys. Res. Commun., 2003, 306, 1042-1049.

[12] Matsumura, K.; Yagi, T.; Yasuda, K. Differential analysis of cell cycle stability in chlamydomonas using on-chip single-cell cultivation system. Jpn. J. Appl. Phys., 2003, 42, L784 - L787.

[13] Moriguchi, H.; Wakamoto, Y.; Sugio, Y.; Takahashi, K.; Inoue, I.; Yasuda, K. An agar-microchamber cell-cultivation system: flexible change of microchamber shapes during cultivation by photothermal etching. Lab Chip, 2002, 2, 125-30.

[14] Hattori, A.; Moriguchi, H.; Ishiwata, S.; Yasuda, K. A 1480/1064 $\mathrm{nm}$ dual wavelength photo-thermal etching system for non-contact three-dimensional microstructure generation into agar microculture chip. Sens. Actuators B., 2004, 100, 455-462.

[15] Sugio, Y.; Kojima, K.; Moriguchi, H.; Takahashi, K.; Kaneko, T.; Yasuda, K. An agar-based on-chip neural-cell-cultivation system for stepwise control of network pattern generation during cultivation. Sens. Actuators B., 2004, 99, 156-162.

[16] Moriguchi, H.; Takahashi, K.; Sugio, Y.; Wakamoto, Y.; Inoue, I.; Jimbo, Y.; Yasuda, K. On-chip neural cell cultivation using agarose-microchamber array constructed by a photothermal etching method. Electrical Eng. Jpn., 2003, 146, 37-42.

[17] Suzuki, I.; Sugio, Y.; Jimbo, Y.; Yasuda, K. Individual-cell-based electrophysiological measurement of a topographically controlled neuronal network pattern using agarose architecture with a multielectrode array. Jpn. J. Appl. Phys., 2004, 43(3B), L403-L406.

[18] Suzuki, I.; Sugio, Y.; Jimbo, Y.; Yasuda, K. Modification of a neuronal network direction using stepwise photo-thermal etching of an agarose architecture. J. Nanobiotechnol., 2004, 2, 7.

[19] Kojima, K.; Moriguchi, H.; Hattori, A.; Kaneko, T.; Yasuda, K. Two-dimensional network formation of cardiac myocytes in agar microculture chip with $1480 \mathrm{~nm}$ infrared laser photo-thermal etching. Lab Chip, 2003, 3, 299-303.

[20] Kojima, K.; Kaneko, T.; Yasuda, K. Stability of beating frequency in cardiac myocytes by their community effect measured by agarose microchamber chip. J. Nanobiotechnol., 2005, 3, 4 .

[21] Yasuda, K.; Okano, K.; Ishiwata, S. Focal extraction of surfacebound DNA from a microchip using photo-thermal denaturation. Biotechniques 2000, 28, 1006-1011.

[22] Curtis, A.S.G.; Wilkinson, C.D.W. Topographical control of cells. Biomaterials 1997, 18, 1573-1583.

[23] Clark, P.; Connolly, P.; Curtis, A.S.G.; Dow, J.A.T.; Wilkinson, C.D.W. Topographical control of cell behaviour. I. Simple step cues. Development 1987, 99, 439-448.

[24] Clark, P.; Connolly, P.; Curtis, A.S.G.; Dow, J.A.T.; Wilkinson, C.D.W. Cell guidance by ultrafine topography in vitro. J. Cell Sci., 1991, 99, 73-77.

[25] Brunette, D.M.; Kenner, G.S.; Gould, T.R.L. Grooved titanium surfaces orient growth and migration of cells from human gingival explants. J. Dental Res., 1983, 62, 1045-1048.

[26] Metz, S.; Holzer, R.; Renaud, P. Polyimide-based microfluidic devices. Lab Chip 2001, 1, 29-34.

[27] Branch, D.W.; Wheeler, B.C.; Brewe,r G.J.; Leckban, D.E. Longterm maintenance of patterns of hippocampal pyramidal cells on substrates of polyethylene glycol and microstamped polylysine. IEEE Trans. Biomed. Eng., 2000, 47, 290-300.

[28] Branch, D.W.; Wheeler, B.C.; Brewer, G.J.; Leckband, D.E. Longterm stability of grafted polyethylene glycol surfaces for use with microstamped substrates in neuronal cell culture. Biomaterials 2001, 22, 1035-47. 
[29] James, C.D.; Turner, J.; Shainm, W. Aligned microcontact printing of micrometer-scale poly-L-Lysine structures for controlled growth of cultured neurons on planar microelectrode arrays. IEEE Trans. Biomed. Eng., 2000, 47, 17-21.

[30] Sholl, M.; Offenhausser, A. Ordered networks of rat hippocampal neurons attached to silicon oxide surfaces. J. Neurosci. Methods 2000, 104, 65-75.

[31] Merz, M.; Fromherz, P. Polyester Microstructures for Topographical Control of Outgrowth and Synapse Formation of Snail Neurons. Adv. Mater., 2002, 14. 141-144.

[32] Connolly, P.; Clark, P.; Curtis, A.S.; J.A. Dow, J.A.; Wilkinson, C.D.W. An Extracellular microelectrode Array for monitoring electrogenic cells in culture. Biosens. Bioelectron, 1990, 5, 223-34.

[33] Wilson, R.J.; Breckenridge, L.; Blackshaw, S.E.; Connolly, P.; Dow, J.A.; Curtis, A.S.; Wilkinson, C.D.W. Simultaneous multisite recordings and stimulation of single isolated leech neurons using planar extracellular electrode arrays. J. Neurosci. Methods 1994, 53, 101-10.

[34] Breckenridge, L.J.; Wilson, R.J.; Connolly, P.; Curtis, A.S.; Dow, J.A.; S.E. Blackshaw, S.E.; Wilkinson, C.D.W. Advantages of using microfabricated extracellular electrodes for in vitro neuronal recording. J. Neurosci. Res., 1995, 42, 266-76.

[35] Potter, S.M.; DeMarse, T.B. A new approach to neural cell culture for long-term studies. J. Neurosci. Methods 2001, 110, 17-24.

[36] Sandison, M.; Curtis, A.S.; Wilkinson, C.D.W. Effective extracellular recording from vertebrate neurons in culture using a new type of micro-electrode array. J. Neurosci. Methods 2002, 114, 6371.

[37] Jimbo, Y.; Kawana, A.; Electrical stimulation and recording from cultured neurons using a planar electrode array. Bioelectrochem. Bioenerg., 1992, 29, 193-204.

[38] Jimbo, Y.; Robinson, H.P.C,; Kawana, A. Simultaneous measurement of intracellular calcium and electrical activity from patterned neural networks in culture. IEEE Trans. Biomed. Eng., 1993, 40, 804-810.

[39] Jimbo, Y.; Tateno, T.; Robinson, H.P.C. Simultaneous induction of pathway-specific potentiation and depression in networks of cortical neurons. Biophys. J., 1999, 76, 670-678.

[40] Jimbo, Y.; Robinson, H.P.C. Propagation of spontaneous synchronized activity in cortical slice cultures recorded by planar electrode arrays. Bioelectrochemistry 2000, 51, 107-115.

[41] Jimbo, Y.; Kawana, A.; Parodi, P.; Torre, V. The dynamics of a neuronal culture of dissociated cortical neurons of neonatal rats. Biol. Cybern., 2000, 83, 1-20.

[42] Carlsson, L. In vitro and in vivo models for testing arrhythmogenesis in drugs. J. Intern. Med., 2006, 259, 70-80.
[43] Kannankeril, P.J.; Roden, D.M. Drug-induced long QT and torsade de pointes: recent advances. Curr. Opin. Cardiol., 2007, 22, 39-43.

[44] Thomsen, M.B.; Matz, J.; Volders, P.G.; Vos, M.A. Assessing the proarrhythmic potential of drugs: Current status of models and surrogate parameters of torsades de pointes arrhythmias. Pharmacol. Ther., 2006, 112, 150-170.

[45] Brimecombe, J.C.; Kirsch, G.E.; Brown, A.M. Test article concentrations in the hERG assay: Losses through the perfusion, solubility and stability. J. Pharmacol. Toxicol. Methods, 2009, 59, 29-34.

[46] Takahashi, K.; Tanabe, K.; Ohnuki, M.; Narita, M.; Ichisaka, T.; Tomoda, K.; Yamanaka, S. Induction of pluripotent stem cells from adult human fibroblasts by defined factors. Cell, 2007, 131, 861872.

[47] Takahashi, K.; Yamanaka, S. Induction of pluripotent stem cells from mouse embryonic and adult fibroblast cultures by defined factors. Cell, 2006, 126, 663-676.

[48] Yu, J.; Vodyanik, M.A.; Smuga-Otto, K.; Antosiewicz-Bourget, J.; Frane, J.L.; Tian, S.; Nie, J.; Jonsdottir, G.A.; Ruotti, V.; Stewart, R.; Slukvin, I.I.; Thomson, J.A. Induced pluripotent stem cell lines derived from human somatic cells. Science, 2007, 318, 1917-1920.

[49] Dimos, J.T.; Rodolfa, K.T.; Niakan, K.K.; Weisenthal, L.M.; Mitsumoto, H.; Chung, W.; Croft, G.F.; Saphier, G.; Leibel, R.; Goland, R.; Wichterle, H.; Henderson, C.E.; Eggan, K. Induced pluripotent stem cells generated from patients with ALS can be differentiated into motor neurons. Science, 2008, 321, 1218-1221.

[50] Ebert, A.D.; Yu, J.; Rose, F.F. Jr.; Mattis, V.B.; Lorso, C.L.; Thomson, J.A.; Svendsen, C.N. Induced pluripotent stem cells from a spinal muscular atrophy patient. Nature, 2009, 457, 277-280.

[51] Soldner, F.; Hockemeyer, D.; Beard, C.; Gao, Q.; Bell, G.W.; Cook, E.G.; Hargus, G.; Bla,k A.; Cooper, O.; Mitalipova, M.; Isacson, O.; Jaenisch, R. Parkinson's disease patient-derived induced pluripotent stem cells free of viral reprogramming factors. Cell, 2009, 136, 964-977.

[52] Suemori, H.; Yasuchika, K.; Hasegawa, K.; Fujioka, T.; Tsuneyoshi, N.; Nakatsuji, N. Efficient establishment of human embryonic stem cell lines and long-term maintenance with stable karyotype by enzymatic bulk passage. Biochem. Biophys. Res. Commun., 2006, 345, 926-932.

[53] Tanaka, T.; Tohyama, S.; Murata, M.; Nomura, F.; Kaneko, T.; Chen, H.; Hattori, F.; Egashira, T.; Seki, T.; Ohno, Y.; Koshimizu, U.; Yuasa, S.; Ogawa, S.; Yamanaka, S.; Yasuda, K.; Fukuda, K. In vitro pharmacologic testing using human induced pluripotent stem cell-derived cardiomyocytes. Biochem. Biophys. Res. Commun., 2009, 385, 497-502.

(C) Yasuda et al.; Licensee Bentham Open.

This is an open access article licensed under the terms of the Creative Commons Attribution Non-Commercial License (http://creativecommons.org/licenses/by-nc/3.0/) which permits unrestricted, non-commercial use, distribution and reproduction in any medium, provided the work is properly cited. 